\title{
DISCRETE-TIME ROBUST PID AND THREE-TERM CONTROL
}

\author{
J. Ackermann ${ }^{*, 1}$ D. Kaesbauer ${ }^{*}$ N. Bajcinca ${ }^{*, 2}$ \\ * Institute of Robotics and Mechatronics \\ German Aerospace Center \\ Oberpfaffenhofen, Germany
}

\begin{abstract}
It has been shown, that stability regions for PID-controllers in a $\left(k_{D}, k_{I}\right)$ plane for fixed $k_{P}$ are convex polygons. This result allows a simple calculation of the set of all stabilizing PID controllers for a given plant. In the present paper this result is transferred to the case of discrete-time PID controllers or threeterm controllers, where stability with respect to the unit circle or other circles in the $z$-plane must be checked. Since the orientation of the cross section planes for polygonal stability regions does not depend on the plant, it is easy to find the set of all simultaneous stabilizers for several representative plant parameters and to select a robust discrete-time controller from this set. A mass-spring-mass system proposed in (Bernstein and Wie, 1990) as a benchmark control problem for digital robust control is used to illustrate the method.
\end{abstract}

Keywords: Singular Frequencies, PID, Three-Term Control, Robust Control, Discrete-Time Control

\section{INTRODUCTION}

It has been shown (Ho et al., 1998), (Ho et al., 2000), that stability regions of PID controllers with fixed proportional gain consist of convex polygons. The proof was given via a generalization of the HermiteBiehler theorem. An alternative proof was given more recently (Munro and Soylemez, 2000) by calculation of the real-axis intersections of the Nyquist plot. In (Ackermann and Kaesbauer, 2001) a derivation and generalization of this result was given by parameter space analysis. It turns out that for variations of two specific controller parameters (or linear combinations thereof) the eigenvalues can cross the imaginary axis (or a parallel to it) only at singular frequencies, that are determined as the roots of a polynomial. At these singular frequencies the stability boundaries are straight lines that bound the stable convex polygons.

In the present paper it is shown that a similar result holds for circles in the eigenvalue plane. They may

\footnotetext{
1 Referring address for notes is Juergen.Ackermann@ylr.de

2 The author would like to thank DAAD for supporting this work.
}

have arbitrary real center and radius. Of special interest is the unit circle, as it is the Schur-stability boundary for discrete-time systems. It is also shown, that circles and half-planes are the only eigenvalue regions for which polygonal stability regions exist. Other controller structures yield other singular eigenvalue regions, see (Bajcinca, 2001).

A discrete-time equivalent of the PID controller has the transfer function

$$
C(z)=\frac{c_{0}+c_{1} z+c_{2} z^{2}}{\left(z+z_{1}\right)(z-1)}
$$

Its structure follows in the quasi-continuous consideration by applying the rectangular integration rule $\left(s \rightarrow\left(z-T_{z}\right)\right.$ to the ideal PID controller $k_{I} / s+k_{P}+k_{D} s$, resulting in $z_{1}=0$, or by the trapezoidal integration rule $(s \rightarrow 2(z-\mathbf{T}) \neq t+1))$, resulting in $z_{1}=1$. Also the realizable PID controller $k_{I} / s+k_{P}+k_{D} s /\left(1+T_{1} s\right)$ converts by the trapezoidal integration rule to the controller structure (1) with a pole at $z_{1}=2_{1}-T /\left(\mathbb{L} \quad{ }_{1}+T\right)$. The following derivation also holds for a three-term controller with an arbitrary second order denominator polynomial. 

continuous design, but consider the direct design of the three controller terms $c_{0}, c_{1}$ and $c_{2}$. The denominator of the controller is combined with the plant transfer function such that the closed-loop characteristic polynomial is

$$
P(z)=\left(t_{i}\right) \quad C(z)+B_{i}(z), \div 1,2 N
$$

with $C(z)=c_{0}+c_{1} z+c_{2} z^{2} . A_{i}(z)$ and $B_{i}(z)$ describe diferent o perating conditions of the plant. The goal is to design a simultaneous stabilizer $C(z)$ for the plant family. Stabilization may-Babdeilitefer to

with the admissible eigentalue region presented by a smaller circle inside the unit circle. First the stability region for a single representative $z), \quad B(z)$ is constructed in tomographic cross section planes such that the stable region is a convex polygon in each plane. We call'this a polygon slice

It is then shown, that the orientation of the polygon slices depends only on theFetability region and not on the plant representative Therefore it is easy to find the set of alfstimbilitensous by the intersection of convex polygons.

\section{SIOLARFIONES}

The characteristic polynomial

$$
P(z)=(x)\left(c_{0}+c_{1} z+c_{2} z^{2}\right)+B(z)
$$

has a root at $z=+j \eta$ if and only if both the real part $R$ and the imaginary part $I$ of $P(z)$ vanish. Now writeAand $B$ in terms of their real and imaginary parts as

$$
\begin{aligned}
& A f+j \eta)=R_{A}+j I_{A} \\
& B(\tau+j \eta)=R_{B}+j I_{B}
\end{aligned}
$$

and substitute $z=+j \eta$,
Here we do not restrict ourselves to a quasi-

The matrix multiplying $\left[c_{0}, c_{2}\right]^{T^{T}}$ is always singular, i.e. 50 re presents two parallel lines in the $\left(c_{0}, c_{2}\right)$ plane. A solution exist if and only if the two lines become identical, i.e. if

$$
d(\eta)=\operatorname{det}\left[\begin{array}{cc}
R_{A} & -c_{1} I_{A} \eta+R_{B} \\
I_{A} & c_{1} R_{A} \eta+I_{B}
\end{array}\right]=0
$$

$R_{A} A_{A} R \quad B$ and $I_{B}$ are polynomials in $\eta$, therefore $d(\eta)$ is a polynomial in $\eta$. Its roots yield the singular frequencies, for each of them a straight line is obtained as stability boundary. The polygon slice in this case is the $\left(c_{0}, c_{2}\right)$-plane for fixed $c_{1}$.

Consider now again the genera).chesill be analyzed, which stability regions lead to axis-parallel polygon slices in a tramsformed $\left(0, r_{1}, r_{2}\right)$-space that is linearly related with $\left(c_{0}, c_{1}, c_{2}\right)$, i.e.

$$
c=T r \operatorname{det} T \neq 0, \text { and } T \text { real }
$$

or, written in detail,

$$
\begin{aligned}
& c_{0}=t_{11} r_{0}+t_{12} r_{1}+t_{13} r_{2} \\
& c_{1}=t_{21} r_{0}+t_{22} r_{1}+t_{23} r_{2} \\
& c_{2}=t_{31} r_{0}+t_{32} r_{1}+t_{33} r_{2}
\end{aligned}
$$

Without loss of genevality we fix ${ }_{1}$ (Fixing 0 or $r_{2}$ only leads to index permutations). 4 Theads

$$
\begin{aligned}
& {\left[\begin{array}{llrrr}
R_{A} & R_{A A} & \eta R_{A} & \left.{ }^{2}-\eta^{2}\right)-I_{A} \tau & \eta \\
I_{A} & I_{A} \tau+R_{A} \eta & \left.I_{A} t^{2}-\eta^{2}\right)+2 R_{A} \tau \eta
\end{array}\right] \times} \\
& \times\left[\begin{array}{cc}
t_{11} & t_{13} \\
t_{21} & t_{23} \\
t_{31} & t_{33}
\end{array}\right]\left[\begin{array}{l}
r_{0} \\
r_{2}
\end{array}\right]+\left[\begin{array}{c}
R_{B} \\
I_{B}
\end{array}\right]+r_{1} \times \\
& \times\left[\begin{array}{cc}
t_{12} R_{A}+t_{22}\left(R_{A} \tau I_{A} \eta\right)+t_{32}\left(R_{A}\left(\tau^{2}-\eta^{2}\right)-{ }^{2}{ }_{A} \tau \eta\right) \\
t_{12} I_{A}+t_{22}\left(I_{A} \tau-R_{A}\right. & \left.\eta)+t_{32}\left(I_{A} \boldsymbol{L}^{2}-\eta^{2}\right)+2 R_{A} \tau \eta\right)
\end{array}\right] \\
& =\left[\begin{array}{l}
0 \\
0
\end{array}\right]
\end{aligned}
$$

Again the $2 \times 2$ matrix multiplying $\left[r_{0} r_{2}\right]^{T}$ is analyzed for singularity. Calculation of jts determinant shows the nontrivial fact that it fadtorizes into an dependent factor, a factor $\eta$ and a factor that depends $R_{A}\left(c_{0}+c_{1} \tau+c_{2}\left(\tau^{2}-\eta^{2}\right)\right)-I$ 多 $\left.\quad c_{1} \eta+2 c_{2} \tau \eta\right)+R_{B}=0$ only on $T$ and $\tau \quad \eta$ $I_{A}\left(c_{0}+c_{1} \tau+c_{2}\left(\tau^{2}-\eta^{2}\right)\right)+R_{A}\left(c_{1} \eta+2 c_{2} \tau \eta\right)+I_{B}=0$

$$
J=\eta\left(R_{A}^{2}+I_{A}^{2}\right)\left(a\left(\tau^{2}+\eta^{2}\right)+2 b \tau+c\right)
$$

or in matrix notation

$$
\begin{gathered}
{\left[\begin{array}{cc}
R_{A} & R_{A} \tau-I_{A} \eta R_{A}\left(\tau^{2}-\eta^{2}\right)-I_{A} \tau \eta \\
I_{A} & I_{A} \tau+R_{A} \eta I_{A}\left(\tau^{2}-\eta^{2}\right)+2 R_{A} \tau \eta
\end{array}\right] \times} \\
\times\left[\begin{array}{l}
c_{0} \\
c_{1} \\
c_{2}
\end{array}\right]+\left[\begin{array}{l}
R_{B} \\
I_{B}
\end{array}\right]=\left[\begin{array}{l}
0 \\
0
\end{array}\right]
\end{gathered}
$$

The imaginary axis as stability boundary as treated in (Ackermann and Kaesbauer, 2001) follows as a special case with. Then for fixed

$c_{1}$

$$
\left[\begin{array}{cc}
R_{A} & -R_{A} \eta^{2} \\
I_{A} & -I_{A} \eta^{2}
\end{array}\right]\left[\begin{array}{l}
c_{0} \\
c_{2}
\end{array}\right]+\left[\begin{array}{c}
-c_{1} I_{A} \eta+R_{B} \\
c_{1} R_{A} \eta+I_{B}
\end{array}\right]=\left[\begin{array}{l}
0 \\
0
\end{array}\right](5)
$$

with

$$
\begin{aligned}
& a=t_{21} t_{33}+t_{23} t_{31} \\
& b=t_{11} t_{33}+t_{13} t_{31} \\
& c=t_{11} t_{23}+t_{21} t_{13}
\end{aligned}
$$

Clearlyvanishes for arbatrary if

- $\eta=0$, i.e. on the real root bogandary, and

$$
-a\left(\tau^{2}+\eta^{2}\right)+2 b \tau+c=0
$$

For $a=0$ there results a straight line $0=-c / \mathscr{D}$ parallel to the imaginary axis with the special case of the imaginary axis if also $c=0$. 
Note that the equations

$$
\begin{aligned}
a & =t_{21} t_{33}-t_{23} t_{31}=0 \\
\tau_{0} & =-c / \mathscr{b} \neq\left({ }_{11} t_{23}-t_{21} t_{13}\right) / 2\left({ }_{11} t_{33}-t_{13} t_{31}\right)
\end{aligned}
$$

allow for many solutions in the nine unknown elements of $T$. A simple solution is

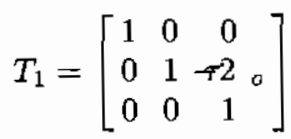

For $\tau_{0}=0$, i.e. the imaginary axis as stability boundary, $T_{1}$ becomes the unit matrix. This case has been handled in (Ackermann and Kaesbauer, 2001).

The only other case ofsanishing describes a circle with raal center

$$
(\tau \rightarrow)^{2}+\eta^{2}=r^{2}
$$

wheren andssatisfy

$$
\begin{array}{ll}
t_{11}=m & t_{21}+\left(r^{2}-m^{2}\right) t_{31} \\
t_{13}=m & t_{23}+\left(r^{2}-m^{2}\right) t_{33}
\end{array}
$$

the $t_{i j}$ must be chosen such that $\operatorname{det} T \neq 0$. A possible choice is

$$
T_{2}=\left[\begin{array}{ccc}
r^{2}-m^{2} & 1 & m \\
0 & 0 & 1 \\
1 & 0 & 0
\end{array}\right]
$$

In terms of the left and right real axis intersections $\tau^{-}=n-$ rand $\tau^{+}=m+r$, the transformation matrix becomes

$$
T_{2}=\left[\begin{array}{ccc}
-\tau^{-} \tau^{+} & 1 & \tau\left({ }^{-}+\tau^{+}\right) / 2 \\
0 & 0 & 1 \\
1 & 0 & 0
\end{array}\right]
$$

\section{FHON S LICS FOR CIRCAR EGLALLELONS}

The previous section has shown that for circles with real center the solution of the poratridns

$I_{P}=0$ is geometrically described by two parallel lines in the $\left(0, r_{2}\right)$-plane with fixed $r_{1}$. A $\Gamma$-stability boundary exists if and only if the two lines become identical. With $T=T_{2}$ of (10) substitutz)d into ( one obtains

$$
\begin{aligned}
& {\left[\begin{array}{ccc}
R_{A} & R_{A} I_{A} & \left.\eta R_{A}{ }^{2}-\eta^{2}\right)+2 I_{A} \tau \eta \\
I_{A} & I_{A} \tau-R_{A} & \left.\eta I_{A}{ }^{2}-\eta^{2}\right)-F_{A} T
\end{array}\right] \times} \\
& \times\left[\begin{array}{cc}
r^{2}-m^{2} & -m \\
0 & 1 \\
1 & 0
\end{array}\right]\left[\begin{array}{l}
r_{0} \\
r_{2}
\end{array}\right]+\left[\begin{array}{c}
R_{B}+R_{A} \\
I_{B}+I_{A}
\end{array}\right]=O(14)
\end{aligned}
$$

Choosing now therpircle (

$$
{ }^{2}+\eta^{2}-r^{2}=0 \text { as }
$$

$\Gamma$-stability boundary we have

$$
\eta=\sqrt{r^{2}-\pi(-i){ }^{2}}
$$

withe $m-r, m+r]$ and the equations in (14) can be written in the form

$$
\begin{aligned}
& A_{1}(\sigma) r_{0}+A_{2}(\sigma) r_{2}+A_{0}(\sigma)=0 \\
& B_{1}(\sigma) r_{0}+B_{2}(\sigma) r_{2}+B_{0}(\sigma)=0
\end{aligned}
$$

The determinant det $\left[\begin{array}{ll}A_{1}(\sigma) & A_{2}(\sigma) \\ B_{1}(\sigma) & B_{2}(\sigma)\end{array}\right]$ vanishes identically. So the equation for the singular frequencies $\sigma=\sigma^{*}$ (the equations in (16) must be proportional) is

$\operatorname{gcd}\left(\right.$ let $\left.\left[\begin{array}{ll}A_{1}(\sigma) & A_{0}(\sigma) \\ B_{1}(\sigma) & B_{0}(\sigma)\end{array}\right], \operatorname{det}\left[\begin{array}{ll}A_{2}(\sigma) & A_{0}(\sigma) \\ B_{2}(\sigma) & B_{0}(\sigma)\end{array}\right]\right)=0$.

The straight line corresponding * is then $\left.\operatorname{Re} P\left(r_{0}, r_{2}\right)\right|_{\sigma=\sigma^{*}}=0$. Additionaliy we have two straight lines corresponding to the intersection points $\tau^{+}$animo $\quad \Gamma$ with the real axis:

$$
\begin{aligned}
& \left.P\left(z_{7} r_{0}, r_{2}\right)\right|_{z=\tau^{+}}=0 \\
& \left.P\left(z, r_{0}, r_{2}\right)\right|_{z=\tau^{-}}=0
\end{aligned}
$$

For each singular frequency resulting form the solution of (18) a straight line is the stability boundary in the $\left(0, r_{2}\right)$-plane. These lines are boundaries of stable (\$stable) polygons.

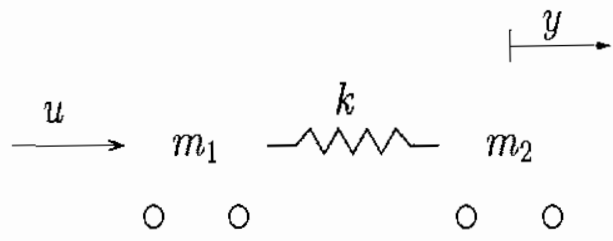

Fig. 1. Schematic diagram of the benchmark problem

\section{EXAMP LE A B ENCHMARK PROBLEM}

\subsection{Escri ption of the system}

In (Bernstein and Wie, 1990) a simple mechanical system is proposed as a benchmark problem for robust controller design. The scheme of the system is shown in Fig. 1. It consistos of two masses 1 and $m_{2}$, which are connected with a spring $k$. The input of the system is the foreacting on the mass 1 , while the output is the positiongof the mass 2 The open-loop transfer function of the system is

$$
G(s)=\frac{k /\left(n l_{1} m_{2}\right)}{s^{2}\left(s^{2}+k \frac{m_{1}+m_{2}}{m_{1} m_{2}}\right)}
$$

The goal consists in robustly stabilizing the system for each operating point inside the parameter uncertainty Q-domain $0.5<k<2$ and $0.5<m_{1}<1$, Fig. 2. We will close the loop by a simple feedback control structure shown in Fig. 3. The sampling rate is chosen to be $T_{s}=0.1 \mathrm{~s}$. 


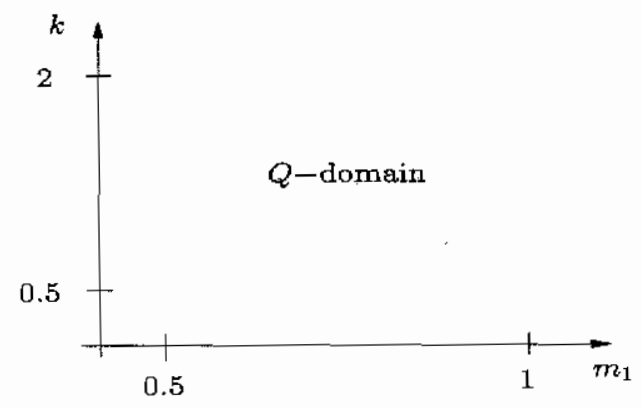

Fig. 2. The unceftaimingin

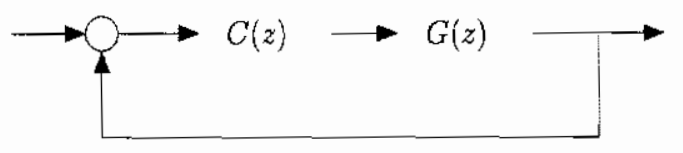

Fig. 3. The control structure

Since the open-loop system has four oscillating modes, the controller structure (1) needs two additional zeros to attract the four eigenvalues into the specified stable region. Therefore we use the following three-term controller structure,

$$
C(z)=10^{4} \frac{\left(z^{2}-1.541 z+0.5992\right)\left(c_{0}+c_{1} z+c_{2} z^{2}\right)}{z(z+0.4047)(z+0.2162)(z-0.4934)}
$$

i.e. we place two zeros left to $z=1$ at $z_{1 / 2}=0.7705 \pm$ $j 0.0744$, and the other two controller zeros remain a matter of design (see next subsection).

\subsection{Schur-stability}

In this subsection we consider the Schur-stability of the system, i.e. we want to find the region of controller parameters such that the eigenvalues of the system remain inside the unit-circle.

A Schur-transformation matrix $T$ has the simple form

$$
T=\left[\begin{array}{lll}
1 & 1 & 0 \\
0 & 0 & 1 \\
1 & 0 & 0
\end{array}\right]
$$

and the new parameter space $r$ is defined by the following equation,

$$
c=T r
$$

with $r_{1}$ being gridded.

In the first step just one operating point from the uncertain Q-domain is considered, e.g. $k=0.5, m_{1}=$ 0.5 . The computation showed that besides the RRB's at $z_{1,2}= \pm 1$, complex singular frequencies (i.e. CRB's) of this operating point live in the interval $-0.2745 \times 10^{29}<r_{1}<12.248$ : a singular frequency in $-0.2745 \times 10^{29}<r_{1}<-0.52236$, three of them in $-0.52236<r_{1}<0$, four singular frequencies in $0<r_{1}<0.00290$ and two singular frequencies in $0.00290<r_{1}<12.248$. Apart from RRB, outside these intervals there are no singular frequencies. The

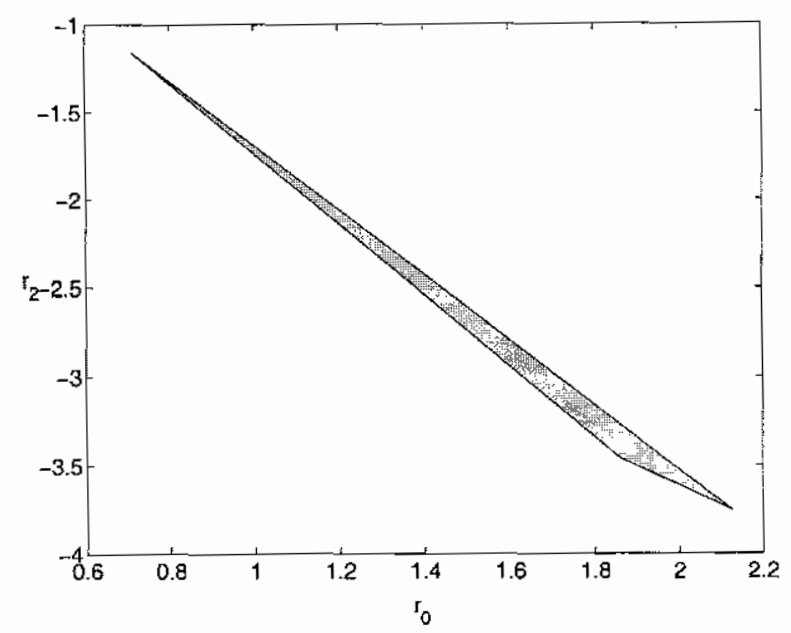

Fig. 4. The stable polygon lying on the plane $r_{1}=$ -0.26118 .

stable polygons, however appear just in the second interval, $-0.52236<r_{1}<0.00290$. For illustration purposes, let us examine the plane $r_{1}=-0.26118$. The corresponding singular frequencies lying on the Schur-circle are,

$$
\begin{aligned}
& z_{1}^{o}=1 \\
& z_{3}^{o}=-0.85435 \pm j 0.51968 \\
& z_{4}^{o}=0.56281 \pm j 0.82656 \\
& z_{5}^{o}=0.91725 \pm j 0.39829 \\
& z_{6}^{o}=-1
\end{aligned}
$$

Each of these singular frequencies generates a singular line on the plane $r_{1}=-0.26118$. The resulting stable polygon is shown in Fig. 4. It is enclosed by the singular frequencies (i.e. lines) $z_{1}^{o}, z_{2}^{o}$ and $z_{6}^{o}$.

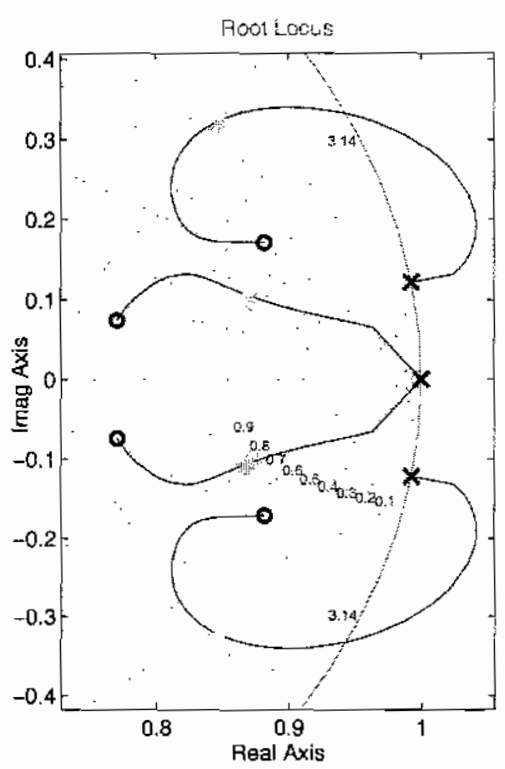

Fig. 5. The root-locus of the feedback system $\left(k_{1}=\right.$ $0.5, m_{1}=0.5$ ) in the vicinity of $z=1$.

In Fig. 5 the new locus $(*)$ of the oscillating modes of the mass-spring-mass is shown. Note that the left zeros were already set in the controller structure, while the right ones are a result of the design procedure. 
We solve further the Schur-stability in the threeparameter space for the operating point $k=0.5, m_{1}=$ 0.5 . The solution is represented by using a tomographic view in the next figure.

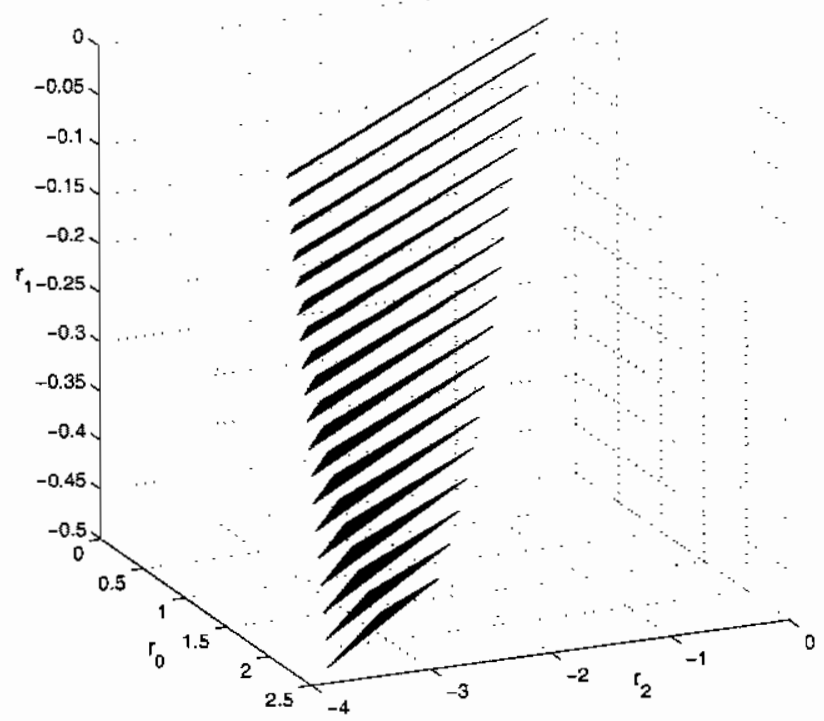

Fig.6 The set of Schur-stable polygons for the operating point $k=0.5, m_{1}=0.5$.

\section{IES OF ROBUST THR E-TRM CONTFE}

Schur-stability does not guarantee satisfactory dynamics of a discrete-time control system. A simple approach would be to keep circles centereneat0 and to reduce the radius from 1 towards zero in order to get a faster decaying envelope of the transients (decaying circles). This approach would however give equal weight to an exponentially decaying solution (positive real pole) and the same solution with alternating sign at each sampling instant (negative real pole). A desirable eigenvalue region that also guarantees enough damping is therefore an additional asymmetric contraction of a satisfactory decaying circle, during which the enter of the circle is shifted to the right as much as possible. An alternative asymmetric contraction of circles is proposed in (Ackermann et al., 199).

For a circle of given center and radius the direction of the polygonal slices is fixed independently of the plant.

The design procedure for a robust controller goes through following steps (Ackermann et al., 199):

- Design a simultaneous $\Gamma$-stabilizer for critical (typically edge)s operating points of thQ domain of uncertain parameters.

- Analyzdstadility of the resulting control system by mappingEstability boundaries to the space of uncertain parameters.

- The vertices of the operating domain must be contained instbeble region by construction.

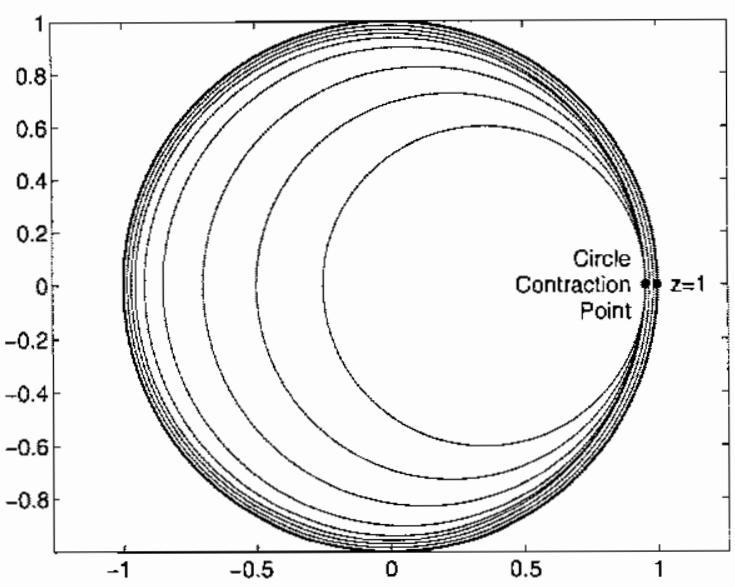

Fig. 7. Symmetric contraction of unit circle followed by asymmetric contraction of decaying circles.

If this is not true for the continuum of admissible operating points, go back to step one with inclusion of additional critical operating points.

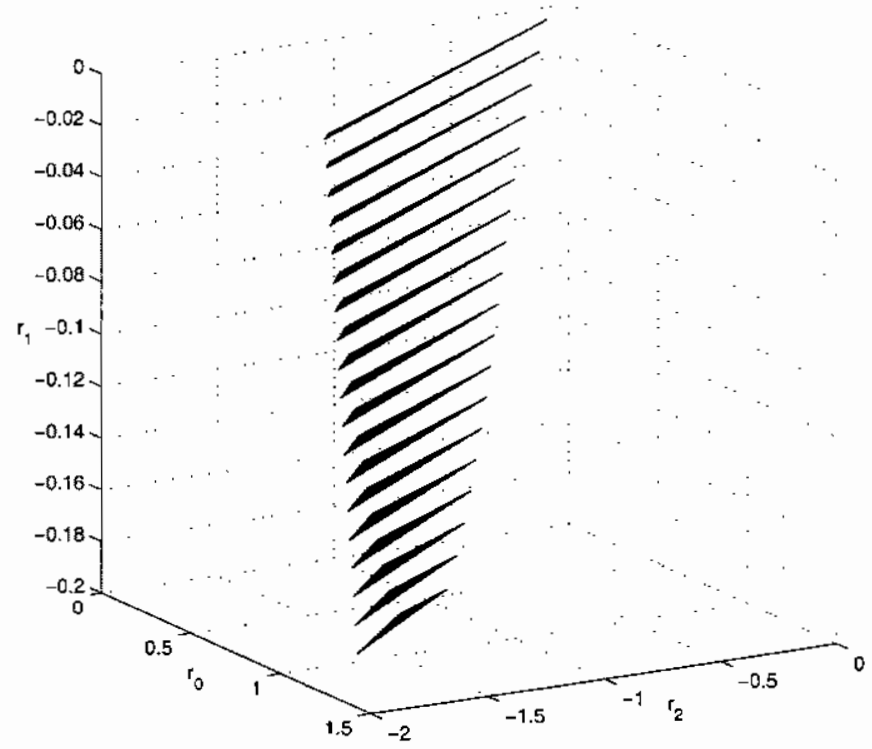

Fig. 8. Simultaneous (robust) Schur-stable region for the four edges of the Q-uncertainty domain.

\section{EXAMP LE (CONBDINU}

\subsection{Robadthastabil ity}

In this article we focus on the first step. In each polygonal slice for fixed $r_{1}$ the simultaneous stabilizers are the intersection of convex polygons, i.e. they are convex polygons, too. We have done this for the Schur-stability in the three-parameter space for the four edgesedfithin of the mass-s pringmass system. The solution is again represented by using a tomographic view in the Fig. 8. Note that the region of robust Schur-stable controllers is (as expected) reduced compared to that of the Schurstable controllers for just the left edge below. 


\subsection{Robust $\Gamma$-stability}

The investigationfereith dircle s

pecifications have shown that transients with the decaying circle of the radius 0.99 are possible. We fixed the right end of thiE-circle and contracted the circle towards it.

No better damping than that guaranteed by the circle with left real axis intersection at -0.89 was possible. A circle with center $m=0.05$ and radtus $\quad .94 \mathrm{re}$ sults. This guaranteerastability with $\sigma_{0}=$ -0.1 and damping $D=0.04$ The stable polygons lie within the interval $-0.000088<r_{1}<0.00252$. In

Fig. 9 we show the spectrum of eigenvalues for the four operating-points corresponding to the controller $r_{0}=0.39713, r_{1}=-0.000119, r_{2}=-0.7805$. Note that this controller pushes the eigenvalues of the operating-points into Eaircle.

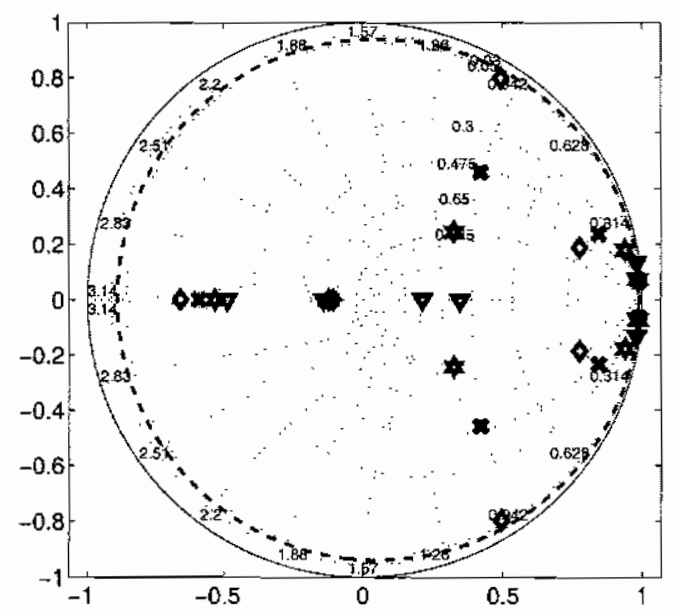

Fig. 9. The eigenvalues of the four uncertainty vertices of mass-spring-mass system.

Finally for a grid on 1 the tomographic view of all simultane $\mathrm{Gt}$ tabilizers is generated.

\subsection{Afal note}

After designing the controller in $r$-parameter space a step of back-transformation of the controller parameters to $c-$ space, by

$$
c=T r
$$

could be done. However it is not trivial and really not necessary to map back the polygons. Instead a controller should be chosen in $r$-space and then back-transformed to $r$-space using. ( 2

\section{COMIONS}

Discrete-time controllers with a free numerator polynomial $c_{0}+c_{1} z+c_{2} z^{2}$ are designed. A tomographic view of the set of all stabilizing controllers would be fficult to com pute if $c_{0}$ or $c_{1}$ or $c_{2}$ is gridded. By appropriate choice of a gridding parameter $r_{1}$, that depends linearly on $c_{0}, c_{1}$ and $c_{2}$, however, the

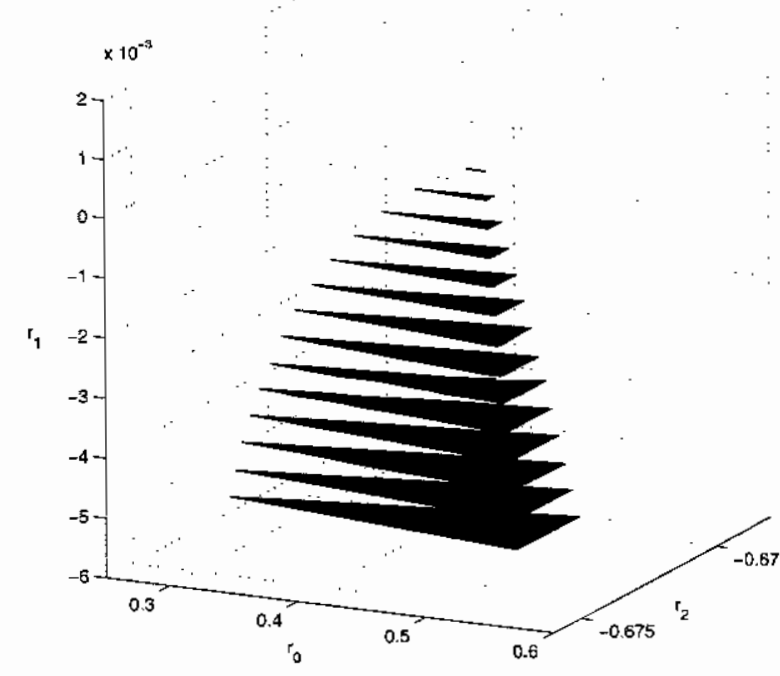

Fig. 10. The Estaltutst polygons for $\Gamma$ the circle with $=0.04$ and $\quad 0.94$ Note that for convenient depiction purposes the polygons are rotated around an axis which passes through $r_{0}=0.330, r_{2}=-0.554$ and is parallel to $\mathrm{I}$ with an anfle of -.95 .

cross section slices show convex polygons as stability regions. Since the 1 -direction does not depend on the plant, simultaneous stabilization for several operating points becomes feasible by intersecting convex polygons.

It is shown that this nice geometric property holds for all circular eigenvalue regions with real center. The design is illustrated by a benchmark robust control example.

\section{RHALAS}

Ackermaln $\mathrm{A}$. Bartlett, D. Kaesbauer, W. Sienel and R. Steinhauspre (199 Robust Control, Systemith uncertain physical parameters. Springer, Berlin.

Ackermalnm, D. Kaesbauer (2001). Stable polyhedra in parameter space. sub. in Automatica.

Bajcinca, N. (2001). The method of singular frequencies for robust defingen in an a parameter space 9 th Mediterranean Conference on Control and Automation, Dubune .

Bernstein, D. and B. Wie (1990), A benchmark problem for robust controller Bynthesis. roc. American Control Conference, San Digo, CA.

Ho, M. T., A. Datta and S. P. Bhattacharryya (1998). Design of P, PI and PID controllers for interval plants $P$ roc. American Control Conference.

Ho, M. T., A. Datta and S. P. Bhattacharryya (2000). Structure and Synthesis of PID Controllers. Springer, London.

Munro, N. and M. T. Soylemez (2000). Fast calculation of stabilizing PID controllers for uncertain parameter systems. roc. SalFA C Symposium on Robust Control Degin, Prague. 